\title{
A ULX and a giant cloud collision in M 99
}

\author{
Roberto Soria ${ }^{1}$ and Diane Sonya Wong ${ }^{2}$ \\ ${ }^{1}$ Harvard-Smithsonian CfA, 60 Garden st, Cambridge, MA 02138, USA \\ ${ }^{2}$ Astronomy Dept, 601 Campbell Hall, Univ. of Cal. at Berkeley, CA 94720-3411, USA \\ email: rsoria@cfa.harvard.edu,dianew@astron.berkeley.edu
}

\begin{abstract}
The Sc galaxy M99 in the Virgo Cluster has been strongly affected by recent tidal interactions, responsible for an asymmetric spiral pattern and a high star formation rate $\left(\sim 10 M_{\odot} \mathrm{yr}^{-1}\right)$. We studied the galaxy with $X M M-N e w t o n$, Keck and the Very Large Array (VLA). The inner disk is dominated by hot plasma with a total X-ray luminosity $\approx 10^{41} \mathrm{erg}$ $\mathrm{s}^{-1}$. At the outskirts of the galaxy, away from the main star-forming regions, there is an ultraluminous X-ray source (ULX) with an X-ray luminosity $\approx 2 \times 10^{40} \mathrm{erg} \mathrm{s}^{-1}$ and a hard spectrum (power-law photon index $\Gamma \approx 1.7$ ). This source is close to the location where a massive H I cloud appears to be falling onto the M 99 disk at a relative speed $>100 \mathrm{~km} \mathrm{~s}^{-1}$. The infalling gas may have been stripped from the nearby "dark galaxy" candidate VIRGOHI 21. We speculate there may be a relation between collisional events, infall of metal-poor gas clouds, and ULX formation.
\end{abstract}

Keywords. X-rays: galaxies - radio lines: galaxies - galaxies: individual (NGC 4254) - X-rays: binaries - black hole physics

\section{Why M99 is an interesting galaxy}

The Sc galaxy M99 (NGC 4254; $d \approx 17 \mathrm{Mpc}$ ) is the brightest spiral in the Virgo Cluster $\left(M_{B}=-20.8\right)$. It has a kinematic mass $\approx 10^{11} M_{\odot}$ (Vollmer et al. 2005; Phookun et al. 1993). It shows unusual features such as a lopsided spiral-arm structure, an H I gas tail and a string of $\mathrm{H}$ I clouds around the stellar disk; the total gas mass in those extra-disk gas structures is $\approx 2 \times 10^{8} M_{\odot}$ (Phookun et al. 1993). This suggests tidal disruptions over the last $\sim 300 \mathrm{Myr}$, due to a close encounter with another Virgo Cluster galaxy, or simply to the cluster potential. The gas clouds around M99 could have been ram-pressure or tidally stripped from the edge of its own gas disk; or they could come from an interacting galaxy. One such nearby object is the "dark galaxy" candidate VIRGOHI 21, a large H I cloud, with a gas mass $\approx 2 \times 10^{8} M_{\odot}$ but without any associated stars, $\approx 120 \mathrm{kpc}$ to the north-west of M 99 (Davies et al. 2004).

Gas-rich galaxies with recent tidal interactions tend to have very high star formation rates (SFRs), and M 99 is no exception, with a rate $\sim 10 M_{\odot} \mathrm{yr}^{-1}$ (Kennicutt et al. 2003). Another phenomenon often associated with tidal interactions and high SFR is the presence of ultraluminous X-ray sources (ULXs). We have used XMM-Newton, VLA and optical data to investigate the X-ray properties of M 99, and the possible connection between ULXs, star formation and collisional events.

\section{Main results of our study}

We summarize here our main results (see Soria \& Wong 2006 for details):

- In the inner galactic disk (at $\lesssim 5 \mathrm{kpc}$ from the nucleus), the X-ray emission is dominated by a soft thermal-plasma component with $k T \lesssim 0.30 \mathrm{keV}$. There is no starburst core. The total unabsorbed luminosity (not including the bright ULX) inside the $D_{25}$ 

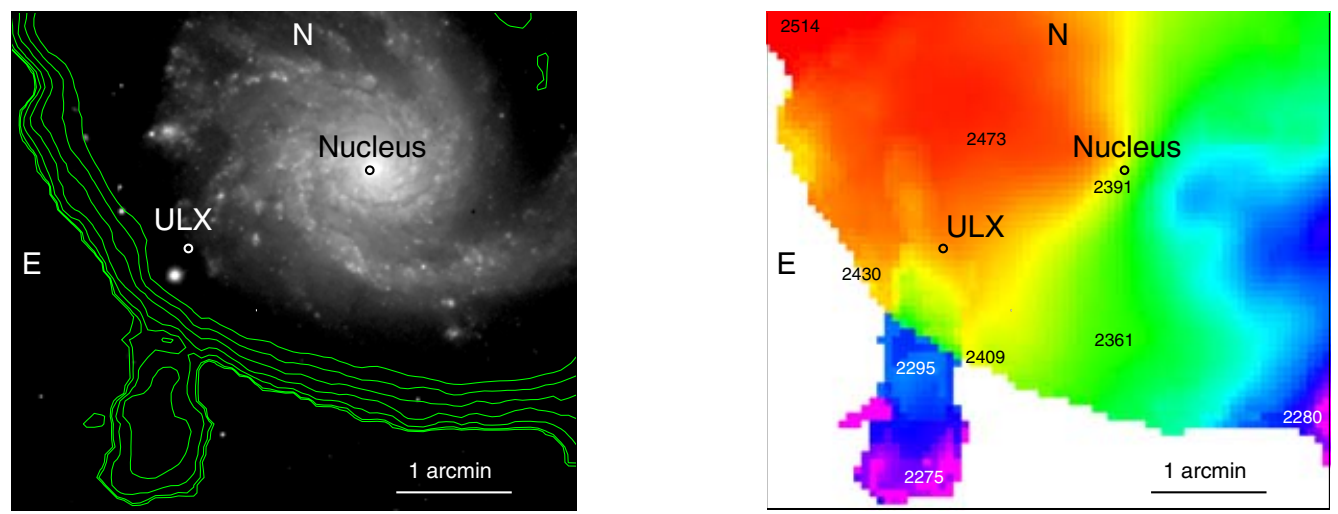

Figure 1. Left panel: H I 21-cm radio flux (from VLA archival data), represented as contours, marking the outer edge of the gas disk; the underlying greyscale image is from VLT/FORS1, in the $R$-band. The projected position of the ULX is well inside the gas disk, but at the edge of the stellar disk, near a large HI cloud. Right panel: velocity map of the HI 21-cm radio emission. Characteristic radial velocities (in $\mathrm{km} \mathrm{s}^{-1}$ ) are also marked at various locations on the plot. The gas cloud is consistent with tidal debris infalling onto the galactic disk from behind (with respect to our viewpoint), at a projected speed $>100 \mathrm{~km} \mathrm{~s}^{-1}$. It appears as though it is impacting or merging with the disk near the ULX position.

ellipse is $\approx(1.2 \pm 0.2) \times 10^{41} \mathrm{erg} \mathrm{s}^{-1}$ in the $0.3-12 \mathrm{keV}$ band; about $15 \%$ of this is due to resolved or unresolved discrete sources.

- The optical nucleus consists of a bright nuclear star cluster $\left(M_{V} \approx-13.5 \mathrm{mag}\right)$ with an old stellar population. Around it, there are a few smaller, much bluer clusters of young stars (typical $M_{V} \approx-11 \mathrm{mag}$ ), much brighter than the nucleus itself in the UV band.

- The bright ULX at the outer edge of the stellar disk has an unabsorbed luminosity of $\approx 2 \times 10^{40} \mathrm{erg} \mathrm{s}^{-1}$ in the $0.3-12 \mathrm{keV}$ band. Its spectrum is well fitted by a powerlaw with photon index $\Gamma \approx 1$.7. Such high/hard states are not uncommon in ULXs but are not generally seen in Galactic BHs. It suggests that we are not seeing the accretion disk directly, and that most of the gravitational power is efficiently transferred to a comptonizing region before being radiated via non-thermal processes.

- There is a massive gas cloud (H I mass $\sim 10^{7} M_{\odot}$ ) seen in projection very close to the ULX. The cloud seems to be falling onto the galactic disk (from behind) with a projected radial velocity $\gtrsim 100 \mathrm{~km} \mathrm{~s}^{-1}$, and perhaps impacting it near the ULX location.

If ULXs are simply a statistical by-product of normal star formation at a sufficiently high rate, it is difficult to explain why there are no ULXs in the inner disk of M 99 despite an SFR $\sim 10 M_{\odot} \mathrm{yr}^{-1}$; instead, the bright ULX is at the outer edge of the stellar disk, where the SFR is orders of magnitude lower. Intriguingly, this ULX seems to be associated with a collisional event between the disk and an infalling gas cloud. The cloud might be made of metal-poor gas stripped from the nearby dark galaxy candidate VIRGOHI 21. We cannot rule out that all this is simply a chance coincidence, but we speculate that there is a physical link between triggered star formation in metal-poor environments and the creation of massive black hole remnants $\left(\sim 30-100 M_{\odot}\right)$ powering ULXs.

\section{References}

Davies, J. et al. 2004, MNRAS, 349, 922

Kennicutt, R. C., et al. 2003, PASP, 115, 928

Phookun, B., Vogel, S. N. \& Mundi, L. G. 1993, ApJ, 418, 113

Soria, R. \& Wong, D. S. 2006, MNRAS, in press (astro-ph/0608648)

Vollmer, B., Huchtmeier, W. \& van Driel, W. 2005, A\&A, 439, 921 